\title{
Introducing form and user concept to Mechanical Engineering Students through Product Design Projects
}

\author{
Qian JI \\ Huazhong University of Science and Technolgoy, \\ Wuhan 430074, China \\ jiqian@mail.hust.edu.cn
}

\author{
Zhou Fang \\ Huazhong University of Science and Technolgoy, \\ Wuhan 430074, China \\ zoeyfangzhou@aol.com
}

\begin{abstract}
This paper discusses a teaching method customized for mechanical engineering students. Based on the educational goals of 'competency-based learning', where students specifically hunt for the skills they need to acquire, a more flexible way of designing has been taught to stimulate their creativity, as well as to complement their capability towards problem solving. In Innovation Design Team (IDT) at Industrial Design Department of Huazhong University of Science and Technology (HUST), year 4 Mechanical Engineering students were introduced to project-based assignments, which were Product Practical and Semantic Analysis, Development of Creative Design on Form and Functionality. The assignments were organized as to transfer their skills from a constructive to a more intuitive and flexible character. Results have shown, through a collection of assignments, how a systematic approach in teaching at the HUST, IDT will efficiently develop the more intuitive and creative skills of students with several years of pure engineering background.
\end{abstract}

Keywords-Industrial Design Skills, Creativity, Form, User

\section{INTRODUCTION}

In an age where economies and industries in China are undergoing tremendous changes, the differences in job scope between engineers and designers are becoming less. More intuitive, emotional and flexible competencies of product design are becoming increasingly important in the future [1]. This has led to a redefinition of the role of higher education of product design to meet the need for the more integrated problem solving.

Nowadays, as the line between engineering and designing are blurred, designers and engineers are expected to cross over into each others field of expertise. Then the generation of engineers and designers we educate today should be trained to deal with more integrated approaches in solving design problem, they are expected to be well-rounded and versatile in generating innovative design solutions at systems, product and component level.

The ingredients, which make up an innovative aptitude, are creativity and communication, as well as the ability to generate ideas, undertake research and conduct experiments.

The emphasis in engineering design training has shifted from technological knowledge and skills towards creativity and innovative thinking.
In fast moving societies with an ambition to become knowledge based economies, such as Singapore and China, findings indicated that job requirements of engineers and industrial designers have become more and more interwoven [2].In the past, design engineers focus primarily on solving technical problems or improving the technical performance of a product. However, as user requirements are becoming more complicated and diverse, engineering tasks in the development of a product cannot clearly and simply be defined anymore. The design engineer is expected to be more versatile, being able to consider usability and form issues to be a certain extent.

From a methodological perspective, four stages can be identified in the design and development process of a product: analyzing, designing, engineering and prototyping. The corresponding design skills for next stage are: 1) Analyzing Stage: the abilities in database searches, identifying and providing insight into the qualification of a design etc, 2) Designing Stage: abilities in creativity, innovation, ideation, sketching and drawing, aesthetics, as well as etc, 3) Engineering Stage: specialized engineering knowledge of mechanisms, molding tools, electrical engineering and manufacturing. 4) Prototyping Stage: abilities in model making, operating models, mechanism models. In reference to the four above steps, engineering students have $\mathrm{m}$ been exposed sufficiently even frequently to design knowledge and practice, as described in stage1,3 and 4, however not much emphasis on abilities in creativity, innovation, ideation, sketching and drawing, aesthetics, that is the reason why they usually solve problems within short eyesight and limited scope of solutions. These solutions are mostly generated using a structured approach of combing existing technical sub-solutions [3].

Sketching and designing are playing important roles in designer's work. Vander Lugt has indicated that sketching is a very relevant tool: 1) to support to interpretation and communication of each other's ideas in group activities, 2) to support a re-interpretive cycle in the individual thinking process, and 3) to enhance access to earlier ideas in order to complement the training profile of an engineer. [4].

Introduction has been designed to educate Mechanical Engineering students the basis of Industrial Design, emphasizing on form and usability. Based on the program in IDT at Industrial Design Department of HUST, authors will discuss the teaching methodology, as well as its execution within studio environment, hope to shed light on the 
exploration of a new teaching method for the Mechanical Engineering students.

\section{Teaching Methodology}

Year 4 Mechanical Engineering Students from IDT were subjected to four related minor design assignments and one major project. In the four minor design assignments, students were given the task to visualize 'simple products followed by redesign. Examples of 'simple' products were: a knife, a chair, a pot, etc. The projects were introduced to develop basic 2-D sketching and drawing skills, as well as to train their sensitivity form and usability in connection to the techno-functional aspects of the product.

The ability of creativity is much more emphasized in the major project through interactive communication of creative ideas and concepts, as there are similarities between engineering and industrial design processes in terms of research detailing.

Seminars were conducted in terms of competency and reflective-based teaching, based on progress observations as well as upon request from students, additional instructions were included in this program.

\section{STUDIO PROGRAM}

PD-Intro stretched over semester period of 12 weeks.

\section{A. The First Four Exercises.}

The first 5 weeks, students were given a series of tutorials on drawing, sketching, rendering and layout set up. These tutorials are conducted in conjunction with the first four exercises on Practical, and Semantic analysis and design. In the first two exercises, students had to constructively and accurately draw 'simple' products of their own choices. Re-design was emphasized in the last two exercises.

Initially students encountered difficulties on constructing the drawing. Several iterative exercises had to be made before the final perspective drawings emerged successfully. As engineering students are to following methods, the quality of drawings and renderings had improved significantly in the second two exercises.

\section{B. The Major Project.}

The remaining 7 weeks were allocated to the design and development of a product. The main objectives of this major project were to introduce iterative divergent and convergent thinking among students, as well as creativity in form creation.

Besides 2-D visualization students had to develop 3-D sketch models and a final mock-up at the end of the project.
The difference in method of working between the first four exercises and major project is that, a mass of alternative solutions were required for the latter. Having undergone intensive training in drawing and sketching during the first four exercises, students felt more confident, and were more intuitive, emotional and efficient then they will be competent in designing and producing. Tutoring sessions mainly focused on ergonomic issues and development of the overall form as well as whole integration of its exterior components, such as the knowledge of mechanisms, molding tools, electrical engineering and manufacturing.

While moving into the detailing phase as the final stages of the concept development, sketch models proved to be very useful in gaining a better understanding of the form. Students were also able to compromise their design intent better with a more intuitive, emotional and flexible interaction with the concept took place.

\section{CONCLUSIONS}

As an elective, the focus of PD-Intro course was to:

Enhance student's creativity and innovation through problem solving.

Guide and aid students think visually by engaging them in drawing, sketching and modeling activities.

Introduce user and form sensitivity to students to get more integrated approaches in solving design problem.

The above mentioned objectives were achieved using a new method of intuitive, emotional and competency-based teaching methodology within a flexible studio environment.

Basic visualization skills, such as drawing, sketching, rendering and model making were taught methodologically. This systematic approach was preferred by engineering students, because of their technical background and structured mindset.

Ultimately, a basic structured training in visualization is essential for mechanical engineering students when iteratively generating ideas and concepts.

\section{REFERENCE}

[1] S. Scrivener: International Journal of Design Sciences and Technology, Vol. 10 (2002) No.2, P.25.

[2] J. Heskett: The Design Task Force: A Strategic Review of Design Education and Practice (The Hong Kong Polytechnic University, Hong Kong, 2003), P.79-108.

[3] N. Cross: Engineering Design Methods, Strategies for Product Design (John Wiley,Chichester 1994),P.22-45

[4] R. Vander Lugt: Design Studies, Vol. 26(2005) No.2, P101-122 